Utah State University

DigitalCommons@USU

Space Dynamics Lab Publications

Space Dynamics Lab

$1-1-2006$

\title{
SOFIE Instrument Overview
}

Andrew Shumway

Chad Fish

Jim Peterson

Peter Mace

James Cook

Follow this and additional works at: https://digitalcommons.usu.edu/sdl_pubs

\section{Recommended Citation}

Shumway, Andrew; Fish, Chad; Peterson, Jim; Mace, Peter; and Cook, James, "SOFIE Instrument Overview" (2006). Space Dynamics Lab Publications. Paper 117.

https://digitalcommons.usu.edu/sdl_pubs/117

This Article is brought to you for free and open access by the Space Dynamics Lab at DigitalCommons@USU. It has been accepted for inclusion in Space Dynamics Lab Publications by an authorized administrator of DigitalCommons@USU. For more information, please contact digitalcommons@usu.edu.

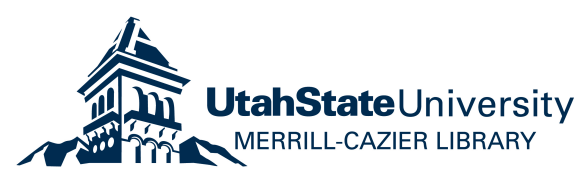




\title{
SOFIE instrument overview
}

\author{
Andrew Shumway ${ }^{1}$, Chad Fish ${ }^{1}$, Jim Peterson ${ }^{1}$, Peter Mace ${ }^{1}$, James Cook ${ }^{1}$, Joel Nelsen ${ }^{1}$, Dale \\ Hooper $^{1}$, Quinn Young ${ }^{1}$, Dr. Steve Wassom ${ }^{1}$, Scott Hansen ${ }^{1}$, John Kemp ${ }^{1}$ (retired) \\ ${ }^{1}$ Space Dynamics Laboratory/Utah State University \\ 1695 North Research Park Way, North Logan, UT 84341 \\ Larry Gordley ${ }^{2}$ \\ Mark Hervig ${ }^{3}$ \\ ${ }^{2}$ Principal Associate, GATS, Inc. \\ 11864 Canon Blvd., Newport News, VA 23606 \\ ${ }^{3}$ GATS, Inc. \\ P.O. Box 449, Driggs, ID 83422
}

\begin{abstract}
Space Dynamics Laboratory (SDL) recently designed, built, and delivered the Solar Occultation for Ice Experiment (SOFIE) instrument as the primary sensor in the NASA Aeronomy of Ice in the Mesosphere (AIM) instrument suite. AIM's mission is to study polar mesospheric clouds (PMCs). SOFIE will make measurements in 16 separate spectral bands, arranged in eight pairs between 0.29 and $5.3 \mu \mathrm{m}$. Each band pair will provide differential absorption limb-path transmission profiles for an atmospheric component of interest, by observing the sun through the limb of the atmsophere during solar occulation as AIM orbits Earth. A pointing mirror and imaging sun sensor coaligned with the detectors are used to track the sun during occulation events and maintain stable alignment of the sun on the detectors.

This paper outlines the mission requirements and goals, gives an overview of the instrument design, fabrication, testing and calibration results, and discusses lessons learned in the process.
\end{abstract}

Keywords: SOFIE, AIM, fabrication, integration, test, calibration, solar, infrared

\section{SCIENCE OVERVIEW}

The primary science objective of the NASA Aeronomy of Ice in the Mesosphere (AIM) mission is to study polar mesospheric clouds (PMCs), also known as noctilucent clouds. Around $83 \mathrm{~km}$ in altitude, they are the highest clouds in our atmosphere and form pole-ward of $50^{\circ}$ latitude in both hemispheres during summer months. Observations to date indicate they are increasing in number and brightness, and are being found closer to the equator. Presently, it is not understood how these clouds are formed, but hypotheses are being made that these clouds may be strongly correlated to global climate stability. Questions are being asked about the relationship between these clouds and global temperature, atmospheric dynamics and chemistry.

The Solar Occultation for Ice Experiment (SOFIE) instrument is specifically designed to measure gasses, temperature, and particles in this cloud layer. SOFIE uses the solar occultation method to measure $\mathrm{H}_{2} \mathrm{O}, \mathrm{CO}_{2}, \mathrm{O}_{3}, \mathrm{CH}_{4}$, and $\mathrm{NO}$ along with temperature and particle extinction at 16 wavelengths [1].

\section{INSTRUMENT REQUIREMENTS}

The AIM satellite will be launched on a Pegasus rocket into a $600-650 \mathrm{~km}$ polar sun-synchronous orbit where it is required to operate for 25 months. SOFIE will perform two solar occultation measurements during each orbit, creating a data volume of $168 \mathrm{Mbits}$ per day. The steering mirror with a $2^{\circ} \mathrm{X} 2^{\circ}$ field-of-regard allows SOFIE to locate and center on the sun. During a solar occultation, SOFIE stares through a $1.8 \mathrm{X} 6$ arc-minute (vertical X horizontal) field at the center of the sun. A sun sensor tracking system references the top edge of the sun to track this position within 15 arc-

Infrared Spaceborne Remote Sensing XIV, edited by Marija Strojnik, Proc. of SPIE Vol. 6297, 62970H, (2006) · 0277-786X/06/\$15 - doi: 10.1117/12.681260 
seconds. With a vertical spatial resolution $<3 \mathrm{~km}$, SOFIE is designed to track the sun from $315 \mathrm{~km}$ down to the hard edge of the Earth.

Sixteen bands pair to form eight channels measuring $\mathrm{H}_{2} \mathrm{O}, \mathrm{CO}_{2}, \mathrm{O}_{3}, \mathrm{CH}_{4}$, and $\mathrm{NO}$, along with particle channels as shown in Table 1. The shorter wavelength bands use silicon carbide and germanium detectors while the longer wavelength bands use mercury cadmium telluride detectors. These bands are spectrally grouped in channel pairs of a strong and weak absorber for the target gas or particles. When a target gas is measured, the strong absorbing band will only detect energy that is mostly attenuated by the gas so that the detector counts drop sharply. On the other hand, weak absorbing band will respond much less dramatically. A third measurement is also processed as the difference between the strong and weak bands. This $\Delta \mathrm{V}$ signal is amplified to preserve high resolution in the difference signal not recoverable by ground post-processing of the strong and weak band signals alone.

Table 1: SOFIE channel overview

\begin{tabular}{|l|l|l|l|l|l|l|l|l|}
\hline Channel & $\mathbf{1}$ & $\mathbf{2}$ & $\mathbf{3}$ & $\mathbf{4}$ & $\mathbf{5}$ & $\mathbf{6}$ & 7 & $\mathbf{8}$ \\
\hline Target Absorber & $\mathrm{O}_{3}$ & particles & $\mathrm{H}_{2} \mathrm{O}$ & $\mathrm{CO}_{2}$ & particles & $\mathrm{CH}_{4}$ & $\mathrm{CO}_{2}$ & $\mathrm{NO}$ \\
\hline Center $\lambda(\mu \mathrm{m})$ & & & & & & & & \\
Strong band & 0.290 & 0.862 & 2.60 & 2.77 & 3.06 & 3.37 & 4.25 & 5.32 \\
Weak band & 0.328 & 1.031 & 2.45 & 2.94 & 3.19 & 3.51 & 4.63 & 4.98 \\
\hline Signal/Noise & $1.0 \mathrm{X} 10^{4}$ & $1.0 \mathrm{X} 10^{6}$ & $2.5 \mathrm{X} 10^{4}$ & $3.0 \mathrm{X} 10^{5}$ & $1.0 \mathrm{X} 10^{5}$ & $4.0 \mathrm{X} 10^{5}$ & $4.0 \mathrm{X} 10^{5}$ & $3.0 \mathrm{X} 10^{5}$ \\
\hline Detector Type & $\mathrm{SiC}$ & $\mathrm{Ge}$ & $\mathrm{HgCdTe}$ & $\mathrm{HgCdTe}$ & $\mathrm{HgCdTe}$ & $\mathrm{HgCdTe}$ & $\mathrm{HgCdTe}$ & $\mathrm{HgCdTe}$ \\
\hline
\end{tabular}

The high signal-to-noise ratios specified in Table 1 drove the design to a $10 \mathrm{~cm}$ diameter telescope and large detector gains in the electronics. The dynamic range of the $\Delta \mathrm{V}$ signal was required to be $2^{13}$ counts. The maximum acceptable nonlinearity for the strong and weak channels was $0.5 \%$ and $1 \%$ for the $\Delta \mathrm{V}$ signals.

\section{INSTRUMENT DESIGN}

SOFIE consists of a separate instrument and electronics box. The instrument is mounted on the top deck of the AIM spacecraft and cables route from the instrument into the spacecraft where there electronics box is mounted to a panel as shown in Figure 1. 

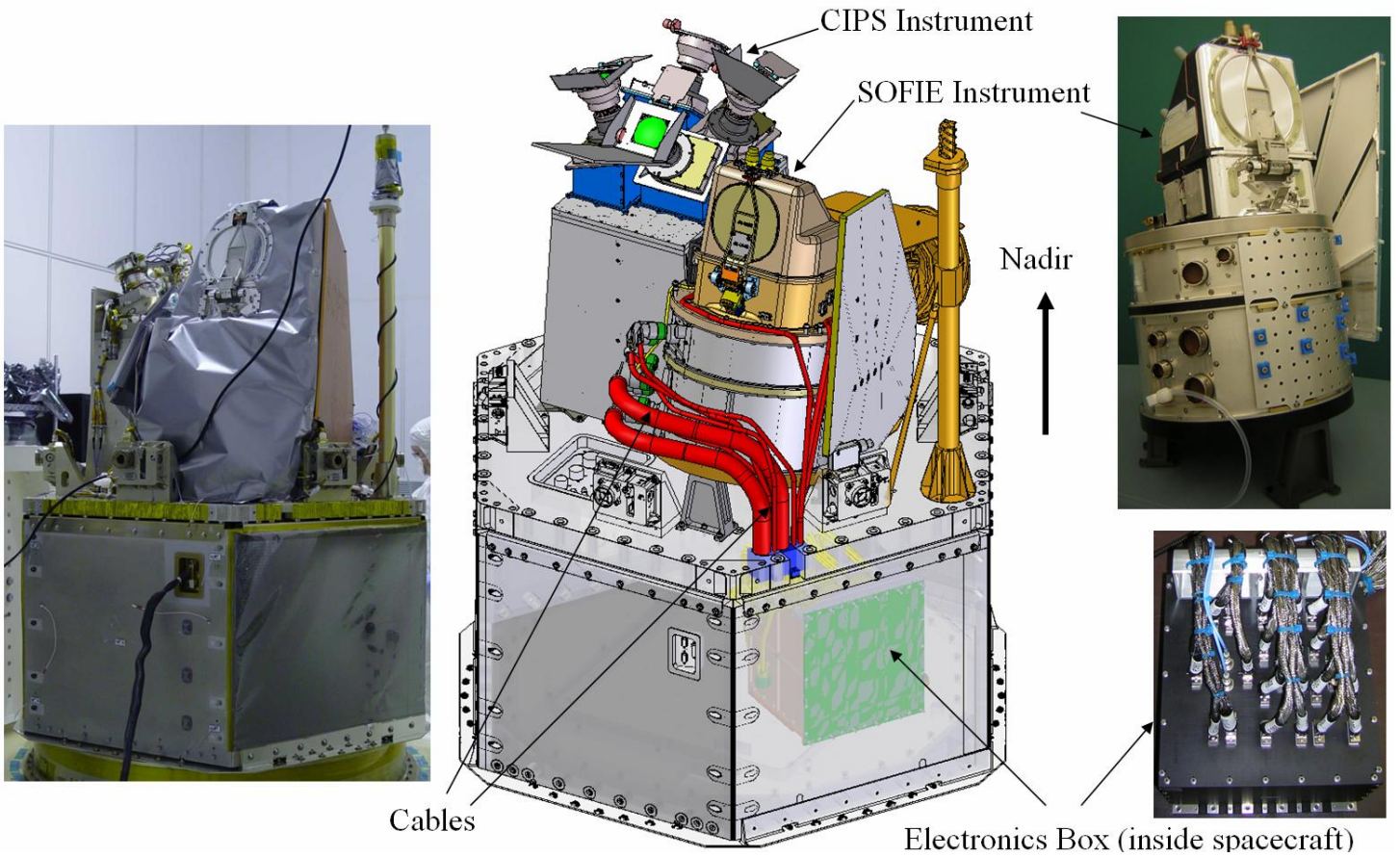

Figure 1: SOFIE instrument and electronics box mounted to the AIM spacecraft

\subsection{Optical-Mechanical}

A two axis steering mirror with a $2^{\circ} \mathrm{X} 2^{\circ}$ degree field of regard capability directs the sunlight into the optical system as shown in Figure 2. A pick-off mirror located above the central obscuration of the telescope, directs the light to the Sun Sensor Module Assembly. After passing through a neutral density and spectral filter the light is focused onto a focal plane array. Processing in the electronics identify the sun, locate the top edge, and direct the optical boresight to a position near the center of the sun for tracking. The algorithms also track jitter and provide feedback to the steering mirror for jitter compensation ${ }^{2}$. 


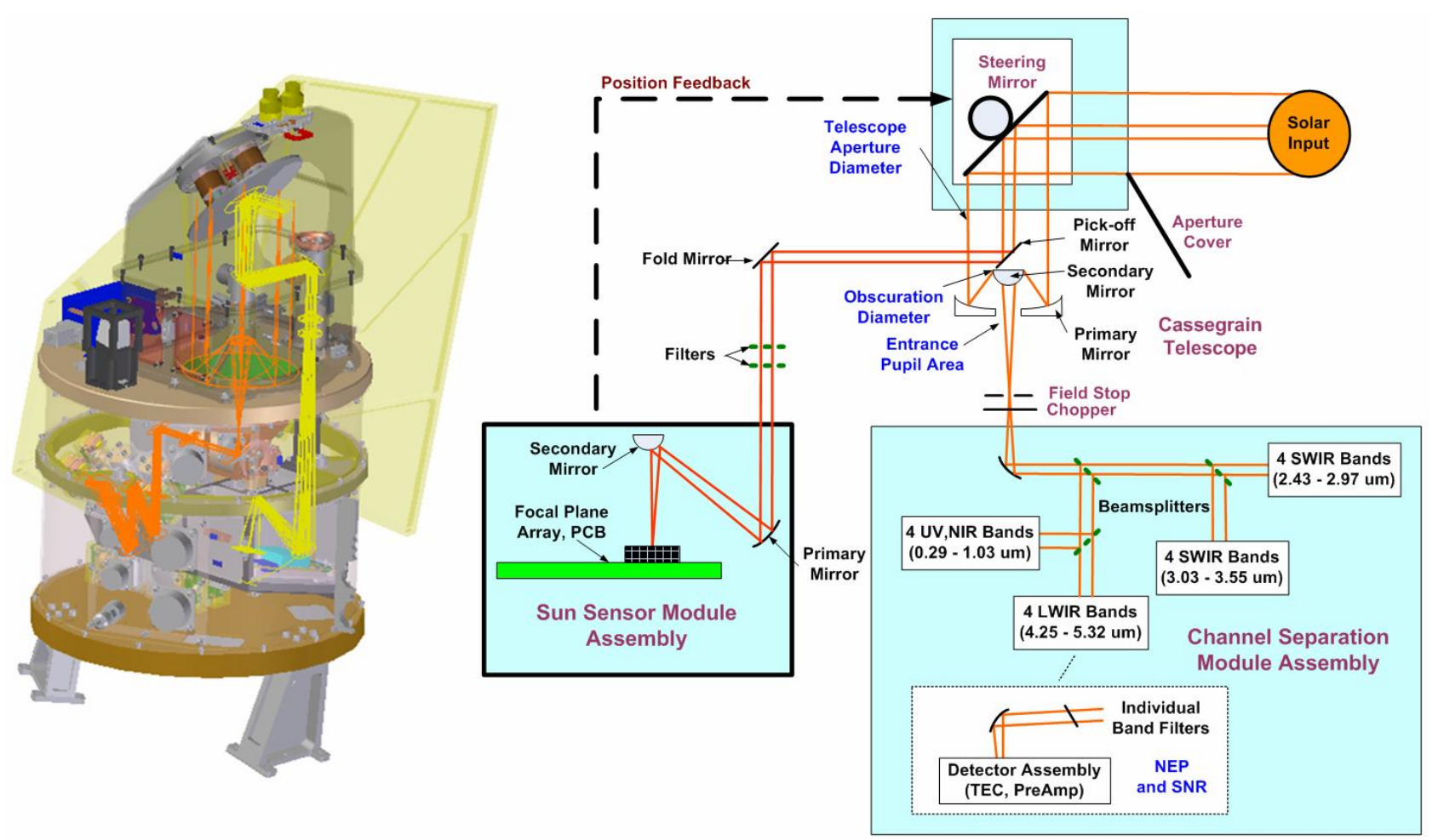

Figure 2: SOFIE instrument optical system

Most of the sunlight strikes the primary mirror shown in Figure 2 and is focused by the cassegrain telescope onto a field stop. The field stop selects the $6 \times 1.8$ arc-minute portion of the sun which passes through a chopper and is then collimated and sent into the channel separation module (CSM). The CSM uses a combination of filters and beam splitters to reflect or transmit spectrally selected portions of the sunlight and relay it to the appropriate band. Using this method, significant energy such as what would be lost using a broadband 50/50\% beam splitter are preserved. As light is concentrated onto the detectors, it is either defocused while the spot underfills the detector, or intentionally overfills the detector. The design of how light was focused on the detectors was driven by the nonlinearity problem encountered during the design. The detectors are cooled by thermo-electric coolers (TECs) to $-40^{\circ}$ and $-65^{\circ} \mathrm{C}$.

The instrument as shown in Figure 3 stands $76 \mathrm{~cm}$ tall, is $46 \mathrm{~cm}$ wide, $58 \mathrm{~cm}$ deep and has a mass of $22.6 \mathrm{~kg}$. The combined mass of instrument, electronics, and cables is $38.6 \mathrm{~kg}$. The steering mirror is mounted in a housing sealed by an aperture cover. A fully redundant set of shape-memory alloy pin-pullers are used for the one-time release of the aperture cover on orbit. Sunlight is reflected toward the telescope mounted at the base of the housing. The CSM and sun sensor module reside together in the body of the instrument. 

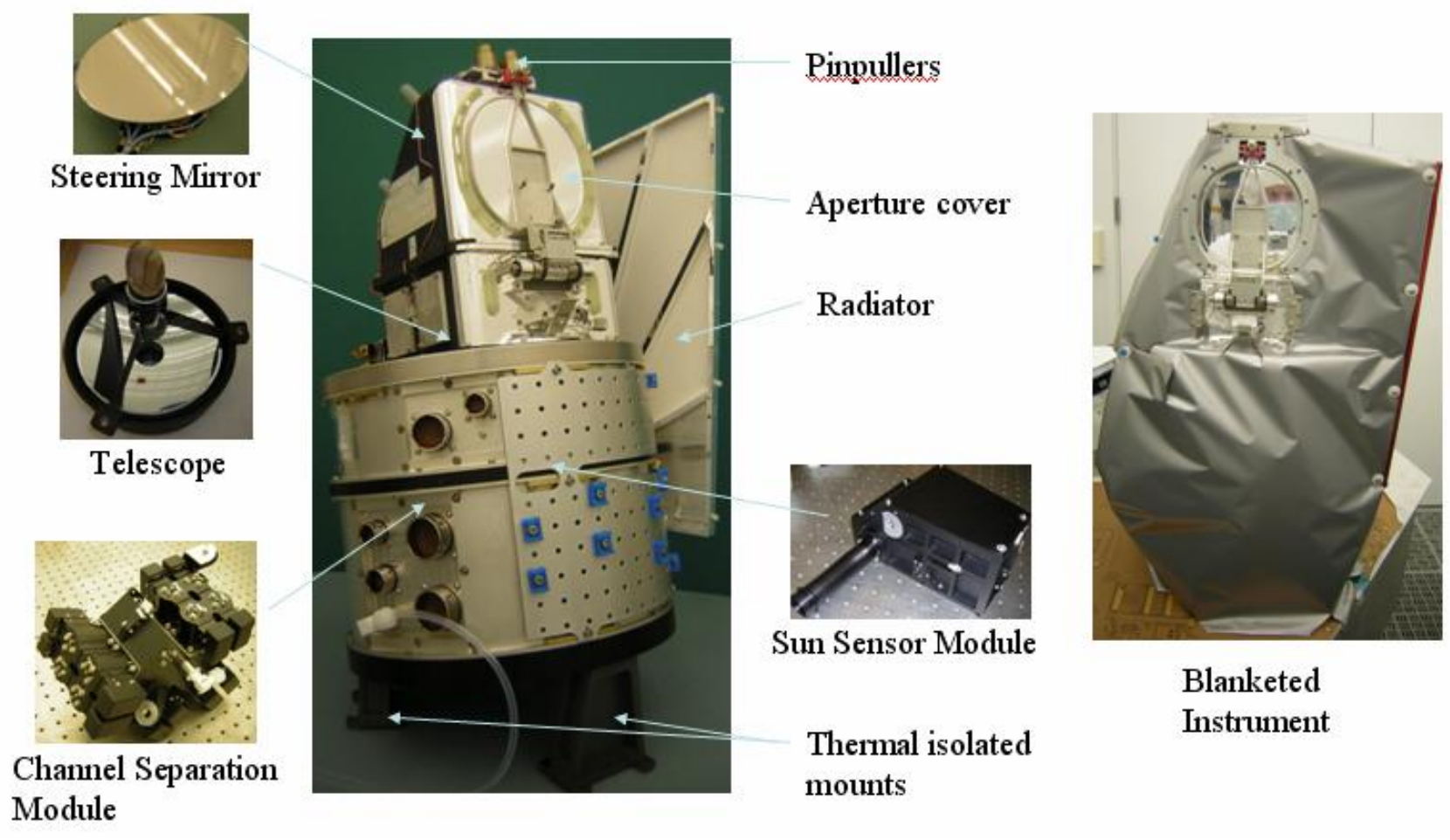

Sun Sensor Module

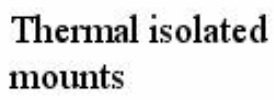

\section{Blanketed}

Instrument

Figure 3: SOFIE Instrument major components

A radiator painted with white Alion Z93C55 electrically conductive paint dissipates the nominally 12 watts of power generated by the thermo-electric coolers and the sun sensor electronics in the instrument. A germanium coated thermal blanket covers the entire instrument and provides electro-static dissipation as well as reasonably low emissivity. The functional temperature range of the instrument is from -50 to $35^{\circ} \mathrm{C}$, but survival heaters are used if needed to keep the sun sensor board and steering mirror position sensor electronics above $-35^{\circ} \mathrm{C}$.

The structure stiffness was designed to be above $100 \mathrm{~Hz}$ with a few exceptions being the bending mode of the radiator and the steering mirror vibration isolation system (VIS). The VIS was added to the design after design loads were increased during the fabrication phase of the instrument. This caused the steering mirror margin from failure to diminish. The VIS was added as a high frequency attenuating support structure allowing the steering mirror to "float" but introducing lower oscillation modes to the design. This compromise was acceptable to the design but caused increased the uncertainty in the boresight direction which will be accounted for during on-orbit operations.

\subsection{Electronics and Software}

The 30 X 30 X $20 \mathrm{~cm}$ electronics box weighs $11.6 \mathrm{~kg}$ and houses ten electronics boards mounted to a backplane with a power conditioning board below. Electronics and software functions include system command and data handling, scan mirror position and stabilization control, communication, signal conditioning, mechanism control, thermal sensors, thermal electric cooler control, housekeeping and power conditioning. The system draws a nominal 46 watts, with peak power of 78 watts. The functional temperature range is from -40 to $+5^{\circ} \mathrm{C}$.

The signal conditioning of the detector channels is accomplished by first using pre-amplifiers behind the detectors to amplify the chopped signals. Signals are then band-pass filtered at the $1000 \mathrm{~Hz}$ frequency of the chopper with a $2 \mathrm{~Hz}$ window. The signals are then synchronously rectified and low pass filtered. Balance attenuators are then used to adjust the gains on each detector. At this point the signal of each detector is fed into a differential amplifier to create the $\Delta \mathrm{V}$ signal and then all three signals are low-pass filtered and digitized. The communications system receives commands from the spacecraft and transmits housekeeping and science telemetry data via a MIL-1553B bus architecture and schedule. 


\section{INSTRUMENT FABRICATION, INTEGRATION, AND TESTING}

The overall fabrication, integration and test flow is shown in Figure 4. Fabrication began first for critical components requiring early testing to prove the design.

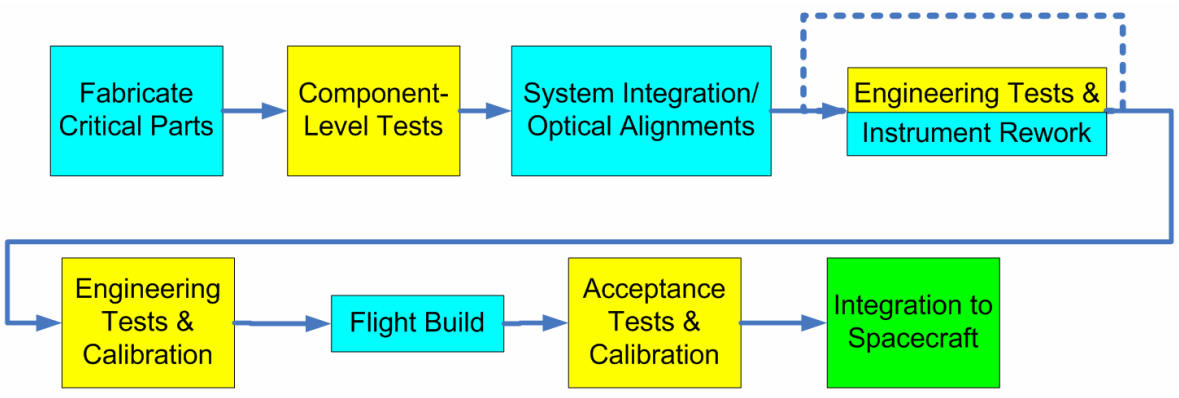

Figure 4: Fabrication, integration and test flow.

\subsection{Component Level Testing}

Component level tests were performed on the aperture cover release mechanism with bench top testing as well as vibration and thermal-vacuum tests. The aperture cover is a mission-critical item and was thus cycled over the qualification temperature range from $-60^{\circ} \mathrm{C}$ to $+55^{\circ} \mathrm{C}$ while deploying the aperture cover at $-55^{\circ} \mathrm{C}$ with each cycle. Testing was also performed on the detector and thermo-electric cooler packages to validate the design of both detector and pre-amp and to gain experience working with these detectors. The tuning fork chopper was also vibration and thermal-vacuum tested as well as lifetime tested. The sun sensor and steering mirror system is a very complex system that required much early testing to validate both the electronics and software. Extensive testing was performed in bench tests using lamps to simulate the sun and a prototype steering mirror. A mass model was built of the instrument structure for tests validating the structural design of the instrument and at the spacecraft level. The mass model was also used for testing of the steering mirror vibration isolation system and later used for a blanket fabrication guide.

\subsection{System Integration \& Optical Alignments}

With continued fabrication of remaining flight parts, system integration began and included optical alignments of the telescope, the sun sensor module, the mid-optics (containing the field stop, chopper, and collimating mirror), the CSM, and the alignment of these assemblies with each other. Both the mid-optics and the cassegrain telescope were aligned using an interferometer at the system focus. The sun sensor module employed a retro-sphere located in the plane of the focal plane and the interferometer was in collimated space. After installation of the sun sensor PCB, a collimated source was presented to this assembly as verification to the optical alignment. The CSM required panning each detector around in the focal plane and mapping out the detector's response to the beam spot. As shown in Figure 5, as the focused spot of light moves across the detector, a flat spot in the curve shows when the spot is fully on the detector. Finally the CSM and sun sensor module were mounted and optically aligned to the telescope assembly.
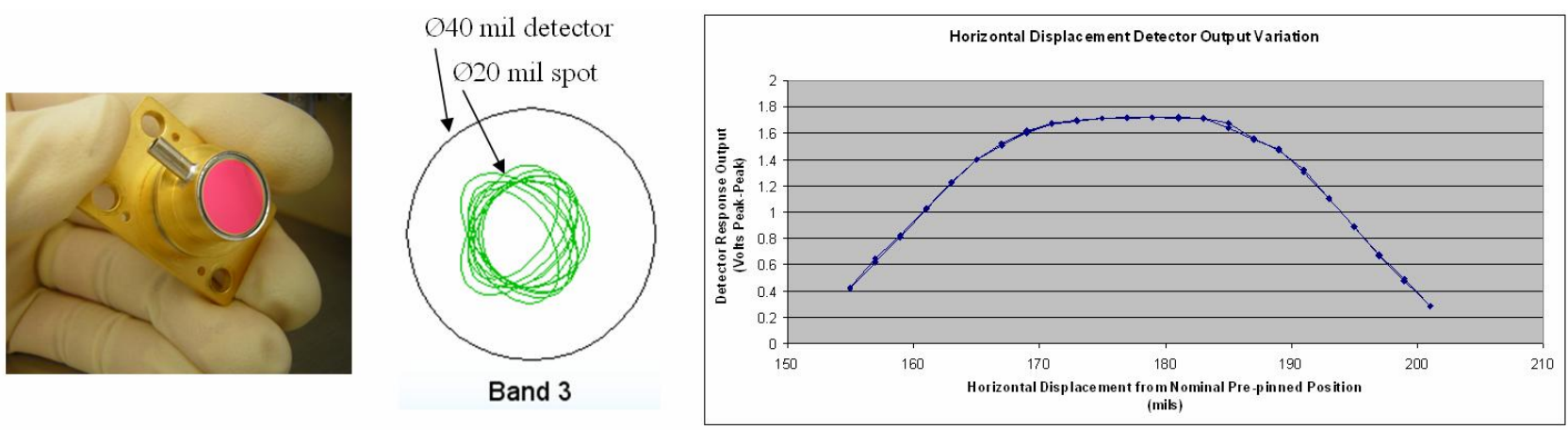

Figure 5: Centering spot on detector 


\subsection{Engineering Tests}

System level engineering tests were then performed on the fully assembled instrument and electronics box. At this time the electronics box was not completely built with final flight parts because measurements and tuning were needed to complete the build. These tests were performed both with the instrument in a clean tent under nitrogen gas purge, and with the instrument and electronics box inside a thermal-vacuum chamber. Temperatures were achieved in the thermalvacuum chamber by surrounding the instrument with a liquid-nitrogen-cooled shroud and using test heaters mounted in three locations on the instrument to simulate heat loads from the sun. The aperture cover was deployed during tests both inside and outside the test chamber.

It was necessary to use the sun as a radiometric source to set the gains of the weak absorbing bands and to test the sun tracking algorithms and system. A sun relay system shown in Figure 6 was developed using an active star tracking gimbal to relay the sun into the laboratory where it could be aligned to the instrument. This allowed critical testing of the SOFIE sun tracking algorithms and system to continue. Because of atmospheric attenuation, the sun was not an adequate source for the strong absorbing bands. A $3000 \mathrm{~K}$ blackbody was thus used to stimulate both strong and weak absorbing bands and transfer the gain settings to the strong absorbing bands as well as adjust the balance attenuators in the electronics. The blackbody also created additional test time for electronics noise and software troubleshooting.

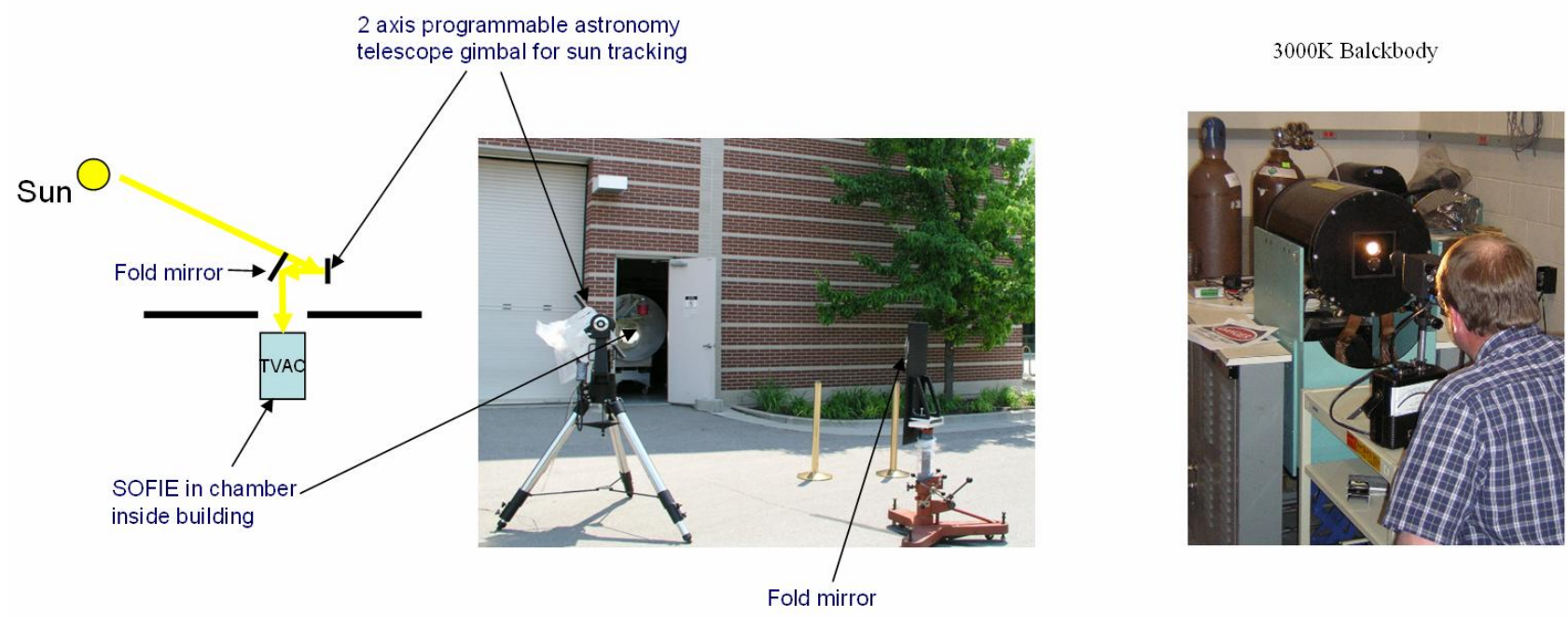

Figure 6: Sun relay system and 3000K blackbody source

A collimator shown in Figure 7 was used to present an interferometer to the instrument for relative spectral responsivity tests. Point source testing was also performed by mapping out the field of view for every detector and determining coalignment of detectors.

During these tests, nonlinearity testing was also performed and was determined to be on the order of $30-50 \%$, which was much larger than initially expected. The cause of the significant nonlinearity was found to be the high irradiance on the detectors. The instrument was thus modified by overfilling some of the detectors and adding a neutral density filter in the optical path. Both modifications reduced the nonlinearity to more reasonable levels below $30 \%$ which could then calibrate out with uncertainties below $1 \%$.

Limited calibration data was collected after the last instrument rework cycle and prior to the final build while this new neutral density filter was yet not installed in the instrument. This configuration enabled the sun and 3000K blackbody sources to stimulate the detectors to higher levels in the dynamic range. At this time, gain and balance attenuator settings were made, and nonlinearity data was collected higher in the dynamic range than otherwise would have been possible. The solution to the nonlinearity problem thus improved the capability to tune and calibrate the instrument. 


\subsection{Acceptance Tests and Calibration}

The final build of SOFIE was performed by first completely disassembling the instrument and electronics. The instrument was precision cleaned for the final time and all the optics were cleaned and inspected. The electronics received their final updates and were conformal coated and baked. During reassembly every fastener was torqued to specification and staked, while all parts and assemblies where photographed and documentation was carefully reviewed and updated. The instrument was placed under a dry nitrogen purge for cleanliness and to protect the detectors from condensation when cooled outside the vacuum chamber.

After performing system functional checkouts, the instrument and electronics box were configured in a near flight-like orientation relative to each other and taken to the electro-magnetic interference test chamber. The system was subjected to a battery of MIL-461C EMI/EMC tests sets.

Vibration tests were next performed on the instrument and the electronics box separately in all three axes. Loads used for instrument testing were $7.7 \mathrm{~g}_{\mathrm{rms}}$ for random vibration, $21.1 \mathrm{~g}$ at $75 \mathrm{~Hz}$ sine burst for Pegasus stage II loads, and $5.7 \mathrm{~g}$ at $10 \mathrm{~Hz}$ sine burst for the Pegasus drop transient. For the electronics box located in a different part of the spacecraft, the loads used for testing were $15.8 \mathrm{~g}_{\mathrm{rms}}$ for random vibration, $15.7 \mathrm{~g}$ at $75 \mathrm{~Hz}$ sine burst for Pegasus stage II loads, and $4.3 \mathrm{~g}$ at $10 \mathrm{~Hz}$ sine burst for the Pegasus drop transient. Sine surveys were used before and after each test to observe possible frequency shifts in the response spectrum. Overall measured frequency shifts were from 0 to $3 \%$ from 0 to $2000 \mathrm{~Hz}$ which met the requirement of $<5 \%$ from 0 to $300 \mathrm{~Hz}$ and $<10 \%$ from 300 to $2000 \mathrm{~Hz}$. After each axis the instrument and electronics box were cabled to each other and an electronics functional testing was performed. For verification of the instrument optical system, theodolite measurements were made of the relative position between the field stop and a reference baffle in the telescope. This optical test showed no relative motion was induced from the vibration tests. In addition, visual inspections showed no sign of structural damage and the subsequent thermal-vacuum test showed no change in thermal performance or detector response.

For thermal-vacuum test planning, temperature states were defined as hot and cold survival, functional, and science temperature limits. The survival limits for the instrument and electronics box respectively ranged from -55 and $-45^{\circ} \mathrm{C}$ to +40 and $+60^{\circ} \mathrm{C}$. Science temperature limits were used to calibrate the instrument at expected on-orbit temperatures with the nominal temperatures being -20 and $+15^{\circ} \mathrm{C}$ for the instrument and electronics box respectively. As with the engineering tests, the blanketed instrument was surrounded by liquid nitrogen cooled shrouds inside in the thermalvacuum chamber as shown in Figure 7. The required temperature states throughout the test were achieved using test heaters mounted in three locations on the instrument to simulate heat loads from the sun while the electronics box was mounted to a temperature controlled plate.
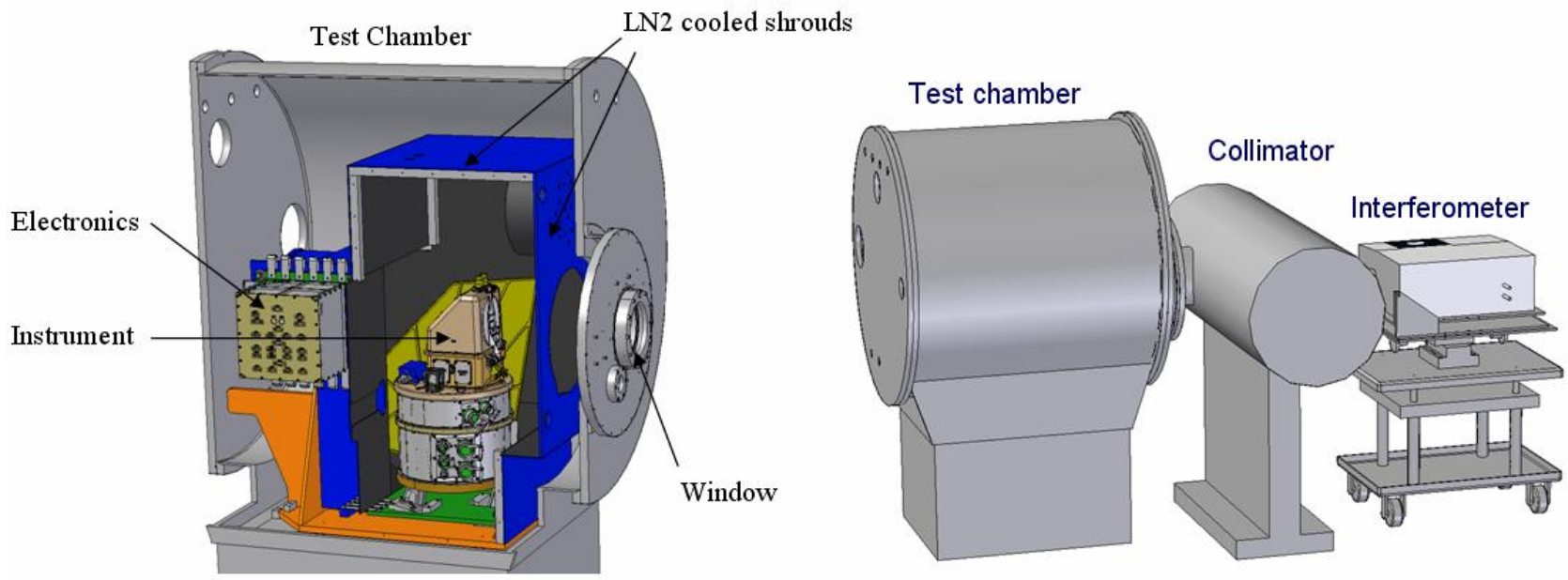

Figure 7: SOFIE instrument and electronics box inside thermal-vacuum test chamber with collimator and interferometer used as source for instrument. 
After an initial vacuum bakeout, the thermal-vacuum testing involved a thermal balance test at various Science temperatures as well as thermal cycle testing between Functional temperature limits. Hot and Cold Survival limits were also reached in an unpowered state. Electrical functional testing was used throughout the thermal balance and thermal cycle periods with required bus voltage levels being testing along with cold transient power cycling and switching between the two redundant bus power supplies for both the electronics and survival heaters. During the thermal balance testing, the aperture cover was deployed to allow calibration data collection to occur simultaneously. Calibration data was collected and Cold, Nominal, and Hot Science temperatures using the same source configurations used during the engineering calibration phase ${ }^{3}$. Thermal balance temperatures and heater power was measured and the survival heaters were tested to ensure they held the Sun Sensor PBC and Steering Mirror position sensor electronics above $-35^{\circ} \mathrm{C}$. Thermal balance data was successfully used to validate the on-orbit thermal models of the instrument and electronics box. First the models were altered to simulate the test chamber environment. After validating and adjusting these models to match the actual measurements, similar adjustments were made to the on-orbit models.

During the thermal-vacuum tests contamination was monitored using several quartz-crystal microbalances (QCM). One was mounted to a port on the instrument where it could see the cavity with the telescope and steering mirror. The other was mounted on the liquid nitrogen cooled shroud where it faced the front of the instrument. Depositions were collected during the bakeout and throughout the thermal-vacuum testing. For the external outgassing, tests show the outgassing rate is a factor of 18 below the external outgassing goal of $3 \times 10^{-11} \mathrm{~g} / \mathrm{cm}^{2} / \mathrm{s}$. For the internal outgassing requirements were driven by potential for photodeposition and darkening of contaminants on the SOFIE steering mirror due to solar UV exposure. Tests show the internal outgassing rate results in only $20 \%$ of the allocated value.

\section{MEASURED INSTRUMENT PERFORMANCE}

While a few minor waivers were required, the electro-magnetic testing, vibration testing, and thermal-vacuum testing showed the instrument and electronics box performed within specification [3, 4]. The sun tracking system which required much design work and testing performed successfully with stability of less than one arc-second. It was able to track and sun through the atmosphere and even in with light clouds or haze interfering with the image. Instrument parameters derived from calibration testing are presented here to illustrate instrument performance.

Measured band limits derived from the relative spectral responsivity (RSR) calibration for all SOFIE bands are shown in Table 2, with specifications shown for comparison [2]. Measured spectral response band edges are within $50 \mathrm{~nm}$ of specification in all cases, and generally much closer than that. RSR measurements included out-of-band measurements to characterize response outside of the intended filter passband. The requirement that out-of-band response provide less than $1 \%$ of the total response is generally met except for bands 1,3 , and 4 . For these bands, the measured out-of-band spectrum will allow correction of measured data to eliminate the impact of excess out-of-band response. Measured spectral response performance for all bands meets science requirements.

Table 2. SOFIE Band Limits

\begin{tabular}{|c|c|c|c|}
\hline \multirow{2}{*}{ Band } & Specification & \multicolumn{2}{|c|}{ Measurement } \\
\cline { 2 - 4 } & $\begin{array}{c}\text { Cuton - Cutoff } \\
(\boldsymbol{\mu} \mathbf{m})\end{array}$ & $\begin{array}{c}\text { Cuton-Cutoff } \\
(\boldsymbol{\mu} \mathbf{m})\end{array}$ & $\begin{array}{c}\text { Cuton-Cutoff } \\
\boldsymbol{\Delta} \text { from spec. } \\
(\boldsymbol{\mu} \mathbf{m})\end{array}$ \\
\hline 1 & $0.2857-0.2941$ & $0.2859-0.2972$ & $0.0002-0.0031$ \\
\hline 2 & $0.3226-0.3333$ & $0.3227-0.3375$ & $0.0001-0.0042$ \\
\hline 3 & $0.8475-0.8772$ & $0.8506-0.8831$ & $0.0031-0.0059$ \\
\hline 4 & $1.0101-1.0526$ & $1.015-1.059$ & $0.0051-0.0068$ \\
\hline 5 & $2.427-2.475$ & $2.436-2.488$ & $0.0087-0.0127$ \\
\hline 6 & $2.577-2.632$ & $2.592-2.643$ & $0.0153-0.0115$ \\
\hline 7 & $2.740-2.794$ & $2.758-2.813$ & $0.0178-0.0190$ \\
\hline 8 & $2.907-2.967$ & $2.910-2.968$ & $0.0034-0.0009$ \\
\hline 9 & $3.030-3.091$ & $3.035-3.093$ & $0.0046-0.0025$ \\
\hline
\end{tabular}




\begin{tabular}{|c|c|c|c|}
\hline \multirow{2}{*}{ Band } & Specification & \multicolumn{2}{|c|}{ Measurement } \\
\cline { 2 - 4 } & $\begin{array}{c}\text { Cuton - Cutoff } \\
(\boldsymbol{\mu} \mathbf{m})\end{array}$ & $\begin{array}{c}\text { Cuton-Cutoff } \\
(\boldsymbol{\mu} \mathbf{m})\end{array}$ & $\begin{array}{c}\text { Cuton-Cutoff } \\
\boldsymbol{\Delta} \text { from spec. } \\
(\boldsymbol{\mu m})\end{array}$ \\
\hline 10 & $3.160-3.226$ & $3.152-3.219$ & $-0.0076--0.0066$ \\
\hline 11 & $3.333-3.401$ & $3.345-3.422$ & $0.0121-0.0212$ \\
\hline 12 & $3.472-3.546$ & $3.445-3.514$ & $-0.0272--0.0322$ \\
\hline 13 & $4.255-4.444$ & $4.220-4.428$ & $-0.0352--0.0163$ \\
\hline 14 & $4.630-4.740$ & $4.581-4.712$ & $-0.0491--0.0280$ \\
\hline 15 & $4.951-5.051$ & $4.959-5.053$ & $0.0080-0.0016$ \\
\hline 16 & $5.263-5.376$ & $5.236-5.396$ & $-0.0275-0.0200$ \\
\hline
\end{tabular}

Instrument non-linearity was characterized using a small attenuator method [3]. Measured full signal non-linearity ranged as high as $29 \%$ for band 7 , although most other bands showed nonlinearity below $10 \%$. Information derived from the non-linearity calibration measurement will allow post-processing of instrument response to produce linear response within the sensor requirement of $0.5 \%$.

SOFIE field-of-view (FOV) characterization yielded results showing performance consistent with instrument specifications of 1.8 arcmin X 6.0 arcmin [3]. Average measured FOV is $1.96 \operatorname{arcmin}$ X $5.10 \operatorname{arcmin}$ (FWHM), FOV band-to-band misalignment is within 0.1 arcmin in the narrow dimension, and within 0.8 arcmin along the long dimension. The SOFIE sensor requirement was simply that FOV misalignment be minimized.

An important assessment of SOFIE instrument performance is instrument off-axis response relative to on-axis response. Instrument specification calls for $99 \%$ of response to be generated within 1 FOV width of FOV center. Measured onaxis response shows performance at specification for 5 bands, while response for other bands is close enough to specification that science performance will not be impacted.

Gain characterization demonstrated a maximum gain error on the order of $25 \%$ for bands $1 \& 2$, while most bands show gain errors of $10 \%$ or less. Gain error refers to the anticipated change in signal gain required to achieve full scale response at maximum illumination on orbit. The SOFIE instrument was designed with adjustable attenuators in the signal path for each band, to allow gains to be balanced within band pairs. The gain errors that were measured are within the range of these balance attenuators.

Background response measurements demonstrated typical background response between 10 and 20 counts for all bands [3]. This result was used to calculate difference-signal $(\Delta \mathrm{V})$ signal-to-noise ratio (SNR) compared to requirement, indicating SNR margin ranging from 1 for bands $15 \& 16$, to approximately 150 for bands 1 and 2 . SNR margin means the system SNR is better than specification by the ratio indicated by the SNR margin.

These parameters and others have demonstrated that the SOFIE sensor performs at or better than specifications that were determined during the experiment design phase. SOFIE is expected to provide the performance necessary to achieve the intended experiment objectives.

\section{LESSONS LEARNED}

Several lessons learned on this project are worth mentioning. The detector nonlinearity problem was a significant surprise and solving it required instrument modifications late in the schedule. It was successfully overcome, but this problem may have been avoided if detector linearity had been examined more closely during the early design phase. The problem was that the detectors exhibit nonlinear behavior with the high irradiance levels used. The solution was to reduce the irradiance and live with the reduced signal to noise. Knowledge gained from this work was also used to improve data from a different NASA instrument already functioning in orbit.

Another problem involved having enough flux during calibration and testing to simulate the sun. We were up against the laws of physics to get more energy out of our sources. Additional component level testing should have been planned 
to help overcome this limitation. The neutral density filter required to solve the nonlinearity problem helped significantly to effectively provide more flux in our system as discussed previously.

We were initially planning on using locking helicoils throughout the system, but found they were a significant and unacceptable source of particulate contamination. We were required to change our design to use non-locking helicoils and then perform staking of the fastener heads using epoxy upon final assembly.

The radiator was painted with white Alion Z93C55 electrically conductive paint. Late in the testing, significant cracking began to propagate on the back side of the radiator. The cause was determined to be significant variations in the paint thickness outside of the recommended thickness. The paint was removed and replaced with ITO coated silverized Teflon tape. The outside of the radiator was also painted with the same paint, but the lack of ribbed structure in the radiator allowed the paint to be applied more uniformly. It thus avoided the flaking problem.

The vibration load specifications given to the SOFIE team from the program were altered several times throughout the design and fabrication process. This caused a late design modification to introduce a vibration isolation system to protect the steering mirror from higher vibration loads. A good method of establishing accurate vibration loads established early in the program would have reduced the potential for significant changes to the instrument late in the schedule.

\section{REFERENCES}

${ }^{1}$ Gordley, L., M. Hervig, J. Russell, C. Fish, G. Paxton, J. Burton, M. McHugh "Sounding the upper mesosphere using broadband solar occultation: The SOFIE experiment,: Proc. of SPIE 6297, 2006.

${ }^{2}$ Wassom, S., C. Fish, M. Whiteley, D. Russak, et al., "SOFIE pointing control system," Proc. of SPIE 6297, 2006.

${ }^{3}$ Hansen, S., C. Fish, D. Romrell, L. Gordley, and M. Hervig, "SOFIE Instrument Ground Calibration," Proc. of SPIE 6297, 2006.

${ }^{4}$ Hansen, S., A. Shumway, J. Peterson, et al., "SOFIE Instrument Model and Performance Comparison," Proc. of SPIE 6297, 2006. 